\title{
Submillimeter Array Observations of Magnetic Fields in Star Forming Regions
}

\author{
Ramprasad Rao*, ${ }^{a}$ Josep M. Girart ${ }^{b}$ and Daniel P. Marrone ${ }^{c}$ \\ a Submillimeter Array, Institute of Astronomy and Astrophysics, Academia Sinica \\ Hilo, HI 96720, USA \\ ${ }^{b}$ Institut de Ciències de l' Espai (CSIC-IEEC) \\ 08193 Bellaterra, Catalunya, Spain \\ ${ }^{c}$ National Radio Astronomy Observatory \& Kavli Institute for Cosmological Physics \\ University of Chicago, Chicago, Illinois, 60637, USA \\ E-mail: rrao@asiaa.sinica.edu.tw
}

\begin{abstract}
The role of magnetic fields in the process of star formation is still not fully understood. Some theories postulate that magnetic fields are important while others state that turbulence is the dominant process and that magnetic fields play a relatively weak role. The recent installation of a polarimetry system at the Submillimeter Array (SMA) has enabled us to conduct observations that could potentially distinguish between the two theories. Some of the nearby low mass star forming regions show hour-glass shaped magnetic field structures that are consistent with theoretical models in which the magnetic field plays a dominant role. However, there are other similar regions where no significant polarization is detected. Ongoing upgrades to the SMA polarimetry system should allow us to make further observations enabling us to increase the sample of observed regions. These SMA measurements will allow us to address observationally the important question of the role of magnetic fields and/or turbulence in the process of star formation.
\end{abstract}

ISKAF2010 Science Meeting - ISKAF2010

June 10-14, 2010

Assen, the Netherlands

\footnotetext{
* Speaker.
} 


\section{Magnetic Fields in Star Forming Regions}

Magnetic fields are believed to play an important role in the star formation process in molecular clouds. The process of star formation is observed to be inefficient, i.e. the rate of star formation is smaller than the value that is predicted by theory. In the absence of any form of support against gravity, the molecular cloud should collapse rapidly on the free-fall timescale. However, observational evidence suggests that the clouds appear to be stable. One agent that could resist gravitational collapse on free-fall timescales is the support provided by magnetic fields. This can explain the observed inefficiency of the star formation process (Mouschovias 2001; Shu et al. 2007). Such a magnetically supported cloud (referred to as a sub-critical cloud) then undergoes flux redistribution through the ambipolar diffusion process. The cloud then is no longer supported by magnetic fields (referred to as supercritical), and collapse starts to occur. The relevant timescale in magnetically regulated star formation is the ambipolar diffusion timescale. If ambipolar diffusion regulated collapse were to occur, then observers should be able to detect ordered, and strong magnetic fields. Theoretical and numerical modeling of the process of gravitational collapse in the presence of magnetic fields predict that the field lines are pinched along the equatorial midplane, producing a morphology that resembles an hour glass. Recently a number of researchers have argued that magnetic fields in the interstellar medium are weak and that star formation is driven by supersonic turbulence (Mac Low 2004). At the boundary layer between two supersonic flows, a density enhancement is created and matter builds up. This enhancement can either decay, or alternatively, it could lead to gravitational collapse of the clump on free fall timescales. It is therefore crucial to be able to measure the structure and morphologies of magnetic fields in star forming regions in order to be able to distinguish between the effects of the competing theories.

One of the ways of detecting magnetic fields is through observations of polarized dust emission from magnetically aligned dust grains. Spinning dust grains in the interstellar medium become aligned such that their short axis becomes parallel to the direction of the magnetic field. The emission produced is parallel to the long axis of the grain. Therefore, the direction of the magnetic field is orthogonal to the direction of the observed polarization. The exact nature of the alignment process is still being studied by a number of theorists. For a long time, it was believed that the alignment produced was by the Davis \& Greenstein (1951) method. Recent results from researchers indicate that the alignment is instead produced by dust grains that are aligned by radiative torques (see review by Lazarian 2007). Previous observations of the polarization conducted at a number of observatories have either been hampered by sensitivity or angular resolution. Emission from dust is strongest at submillimeter wavelengths and observations made by interferometric arrays can produce the detailed maps needed. The Submillimeter Array (Ho, Moran, \& Lo 2004) is the right instrument to conduct such observations as it is not limited by the factors mentioned. The SMA is currently the only interferometer array that can conduct these observations and obtain high resolution polarization and magnetic field maps of a number of such star forming regions.

\section{Observational Techniques and Methodology}

Linear polarization measurements with interferometers are best done with circularly polarized feeds (see Thompson, Moran, \& Swenson 2001). This minimizes the effect of gain errors, as the 
cross-correlation of opposite circular polarizations does not involve the Stokes total intensity (I) parameter. The SMA uses quarter-wave plates to transform its linearly-polarized feeds into circular feeds. The waveplates are made of either quartz or sapphire, with low-density polyethylene antireflection coatings. Most of the dust polarization observations to date have been carried out in the $345 \mathrm{GHz}$ band. The waveplates are tuned for observations at $342 \mathrm{GHz}$ and the expected instrumental polarization performance should allow their use within $20 \mathrm{GHz}$ of this frequency. Since only a single polarization is received at any given time, the left $(\mathrm{L})$ and right $(\mathrm{R})$ polarization states are time-multiplexed on each antenna using a fast Walsh function switching pattern of periodicity 16 in order to sample all possible cross correlations (LL, LR, RL, RR) on every baseline (see Thompson, Moran, \& Swenson 2001 for more information on Walsh functions). The data are averaged over this Walsh cycle to produce quasi-simultaneous dual polarization measurements. Beam smearing resulting from this averaging process is negligible. A brief description of these techniques is provided in Marrone et al. (2006) and a more detailed discussion of the methodology (both hardware and software aspects) is available in Marrone (2006) and Marrone \& Rao (2008).

\section{Observations: Low Mass Star Forming Regions}

The initial targets for the polarization observations were regions of low mass young stellar objects (YSOs). Such regions are somewhat isolated and the maps of the magnetic field are expected to be easier to interpret and model. Some of these observations are discussed in this section.

\subsection{NGC 1333 IRAS 4A}

The first object that was chosen for this study was the star forming region NGC 1333 IRAS 4A. This is a Class 0 protostar which has been resolved into a binary system with a separation of $\sim 1^{\prime \prime}$. These observations, which were conducted by Girart, Rao, \& Marrone (2006), are shown in Figure 1. For the first time, we show that in a low-mass star forming region the observed properties of the magnetic field are in agreement with the standard theoretical models of isolated star formation in magnetized molecular clouds: the magnetic field traces a clear hourglass morphology. On larger scales the polarization direction is quite uniform at a position angle of $\sim 145^{\circ}$ and is in excellent agreement with earlier lower resolution observations. However, on small scales ( $\sim 200 \mathrm{AU})$ the field is significantly distorted or "pinched" and the morphology resembles the hour glass shape that is predicted by theory. The symmetry axis for the magnetic field appears to be approximately perpendicular to the major axis of the extended disk. This method of using the polarized emission to map the magnetic field can only measure the direction of the magnetic field in the plane of the sky. However, by using the Chandrasekhar \& Fermi (1953) method (henceforth referred to as the CF Method) we can indirectly infer the strength of the magnetic field as well. The CF method uses the intrinsic dispersion in the polarization angles to obtain the magnetic field strength. We obtain a plane of sky magnetic field strength of $4 \mathrm{mG}$. Our analysis reveals that the magnetic field is substantially more important than turbulence in the evolution of the NGC 1333 IRAS4A circumbinary envelope. The magnetic field morphology and the mass-to-magnetic-flux ratio indicate that gravity has overcome magnetic support, as predicted for this stage of star formation. The observed misalignment of the magnetic, outflow and envelope axes might have been an important factor in triggering the observed fragmentation of the core, leading to the formation of a binary system. 


\subsection{IRAS 16293}

This star forming region is also a Class 0 object similar in nature to NGC 1333 IRAS 4A that was discussed previously. It is a binary system as well, separated into two components A and B with a projected distance or $\sim 750 \mathrm{AU}$. We find that the large scale global direction of the field, which is perpendicular to the observed polarization, appears to be along the dust ridge where the emission peaks (Figure 1; Rao et al. 2009). On smaller scales we find that the field structure is significantly different for the two components of the binary. The first component, source A, shows a magnetic field structure which is "hourglass" shaped as predicted from theoretical models of low mass star formation in the presence of strong magnetic fields. However, the other component, source B, shows a relatively ordered magnetic field with no evidence of any deformation. Our calculations show that in IRAS 16293 the magnetic energy is stronger than the turbulent energy but is approximately similar to the centrifugal energy. There is considerable misalignment between the outflow direction and the magnetic field axis and this is roughly in agreement with model predictions where the magnetic energy is comparable to the centrifugal energy. In conjunction with other observations of the kinematics as determined from the outflow energetics and chemical differentiation we find that our results provide additional evidence to show that the two protostars appear to be in different stages during their evolution.

\section{Observations: High Mass Star Forming Regions}

The two objects discussed above have both been low mass star forming regions. It appears that in these two regions, the magnetic field dominates over turbulence in the star formation process. We will now present some observations of the magnetic field in a selected sample of high mass star forming regions and show that the morphologies of the magnetic field show interesting structures.

\subsection{G31.41}

G31.41+0.31 is a high mass star-forming region located $7900 \mathrm{pc}$ away. It harbors a massive $\left(\sim 500-1500 \mathrm{M}_{\odot}\right)$, rotating hot molecular core with a luminosity of $3 \times 10^{5} \mathrm{~L}_{\odot}$. The massive ultracompact HII region has not yet developed, so the embedded young stellar objects are in a very early stage of their evolution. The mass of the toroid is much larger than the dynamical mass required for equilibrium, which suggests that the toroid may be gravitationally unstable and undergoing collapse. The spectro-polarimetric observations carried out with the SMA (Figure 2; Girart et al. 2009) in the compact and extended configuration at $345 \mathrm{GHz}$ has allowed us to study the massive hot core at angular resolution slightly below one arcsecond, tracing scales of several thousand AU. The maps clearly show that the magnetic field lines threading the hot core are pinched along the major axis of the core, where the velocity gradient due to rotation is observed, acquiring a hourglass morphology. The emission of the myriad of spectral lines shows two interesting properties. First, the lines with the lowest energy levels (few tens of K), such as the $\mathrm{C}^{34} \mathrm{~S} 7-6$, show an inverse P-Cygni profile, indicative of infall motions. Second, a comparison of the velocity gradient along the major axis for different methanol lines show a smaller rotation velocity in the more spatially compact lines (typically the higher excitation ones). This is indicative that the angular momentum is not conserved during the collapse. The analysis of the SMA data show that the magnetic field 
dominates energetically (with respect to centrifugal and turbulence forces) the dynamics of the collapse, and that there is evidence of magnetic braking.

\subsection{G5.89}

In addition to the G31.41 observations mentioned above, the SMA has also observed the ultracompact HII region G5.89-0.39 (Tang et al. 2009). In this source, the dust polarization is not uniformly distributed in the entire dust core (See Figure 3). Most of the polarized flux is located around the HII ring. The detected polarization vectors can be separated into two groups according to the distribution of the position angle: The vectors belonging to group " $\mathrm{x}$ " are located at the sharp $870 \mu \mathrm{m}$ dust ridge. These vectors are more likely tracing the compressed field. The vectors belonging to group "o" are located at the edge of the sharp dust ridge and are more likely from an extended structure. By using the CF method, the estimated lower limit of the B field strength from the extended component (group "o") is $1.3 \mathrm{mG}$. The B field morphology in G5.89 is clearly disturbed by the expansion of the HII region and the molecular outflows in G5.89. From the analysis of the kinematics by using the $\mathrm{C}^{17} \mathrm{O} 3-2$ line emission and the comparison of the energy density (pressure) from the radiation, $\mathrm{B}$ field and kinetic motions, it is proposed that the $\mathrm{B}$ field structures are already overwhelmed and dominated by the newly formed massive stars.

\section{Polarization in Protoplanetary Discs}

The SMA observations of the polarized emission can also be used to provide some information on the nature and efficiency of the dust grain alignment mechanisms. Previous observations (Tamura et al. 1999) and theoretical predictions (Cho \& Lazarian 2007) have suggested that a polarization fraction of 2-3\% should be commonly observed for protoplanetary disks around young stars. We observe the disks around two nearby stars, HD 163296 and TW Hydrae, using the SMA polarimeter (Figure 4), and detect no polarized emission from either disk. The SMA observations set the most stringent limits to date on the millimeter wavelength polarization from protoplanetary disks, and rule out the fiducial Cho \& Lazarian (2007) model at the 10-sigma level. By comparing our observations to the model predictions of Cho \& Lazarian (2007), we determine that the factors most likely contributing to the suppression of polarized emission relative the fiducial model are the roundness of large grains, inefficient alignment of grains with the magnetic field, and a random "tangled" component to the magnetic field lines.

\section{Summary}

Preliminary results with the SMA thus far appear to indicate that magnetic fields are relatively strong when compared to turbulence in the limited sample of objects observed thus far. We are continuing to use the current polarimetry system to conduct further observations towards other similar objects. In the future, the use of dual polarization receivers will vastly improve the sensitivity and simplify the operation. This will allow us to make such observations towards a larger sample of YSOs enabling us to better understand the role of magnetic fields in the star formation process. 


\section{References}

[1] S. Chandrasekhar, \& E. Fermi, Magnetic Fields in Spiral Arms, ApJ, 118 (1953) 113

[2] J. Cho, \& A. Lazarian, Grain Alignment and Polarized Emission from Magnetized T Tauri Disks, ApJ, 669 (2007) 1085

[3] L. Davis, \& J. L. Greenstein, The Polarization of Starlight by Aligned Dust Grains, ApJ, 114 (1951) 206

[4] J. M. Girart, M. T. Beltrán, Q. Zhang, R. Rao, \& R. Estalella, Magnetic Fields in the Formation of Massive Stars, Science, 324 (2009) 1408

[5] J. M. Girart, R. Rao, \& D. P. Marrone, Magnetic Fields in the Formation of Sun-Like Stars, Science, 313 (2006) 812

[6] P. T. P. Ho, J. M. Moran, \& K. Y. Lo, The Submillimeter Array, ApJ, 616 (2004) L1

[7] A. M. Hughes et al., Stringent Limits on the Polarized Submillimeter Emission from Protoplanetary Disks, ApJ, 704 (2009) 1204

[8] T. R. Hunter, C. L. Brogan, R. Indebetouw, \& C. Cyganowski, Subarcsecond Submillimeter Imaging of the Ultracompact H II Region G5.89-0.39, ApJ, 680 (2008) 1271

[9] A. Lazarian, Tracing magnetic fields with aligned grains, Journal of Quantitative Spectroscopy \& Radiative Transfer, 106 (2007) 225

[10] M. M. Mac Low, \& R. S. Klessen, Control of star formation by supersonic turbulence, Rev. of Modern Physics, 76 (2004) 125

[11] D. P. Marrone, Submillimeter properties of Sagittarius A*: The polarization and spectrum from 230 to $690 \mathrm{GHz}$ and the submillimeter array polarimeter, Ph.D. Thesis, Harvard University, (2006)

[12] D. P. Marrone, J. M. Moran, J.-H. Zhao, \& R. Rao, Interferometric Measurements of Variable 340 GHz Linear Polarization in Sagittarius A*, ApJ, 640 (2006) 308

[13] D. P. Marrone, \& R. Rao, The submillimeter array polarimeter, in proceedings of the SPIE, 7020 (2008) 70202B

[14] T. Mouschovias, Star Formation Models with Magnetic Fields, in ASP Conference Proceedings, 248 (2001) 515

[15] R. Rao, J. M. Girart, D. P. Marrone, S. P. Lai, \& S. Schnee, IRAS 16293: A “Magnetic” Tale of Two Cores, ApJ, 707 (2009) 921

[16] F. H. Shu, D. Galli, S. Lizano, M. J. Cai, Magnetization, accretion, and outflows in young stellar objects, IAU Symposium, 243 (2007) 249

[17] M. Tamura et al., First Detection of Submillimeter Polarization from T Tauri Stars, ApJ, 525 (1999) 832

[18] Y. W. Tang et al., Evolution of Magnetic Fields in High Mass Star Formation: Submillimeter Array Dust Polarization Image of the Ultracompact H II Region G5.89-0.39, ApJ, 695 (2009) 1399 

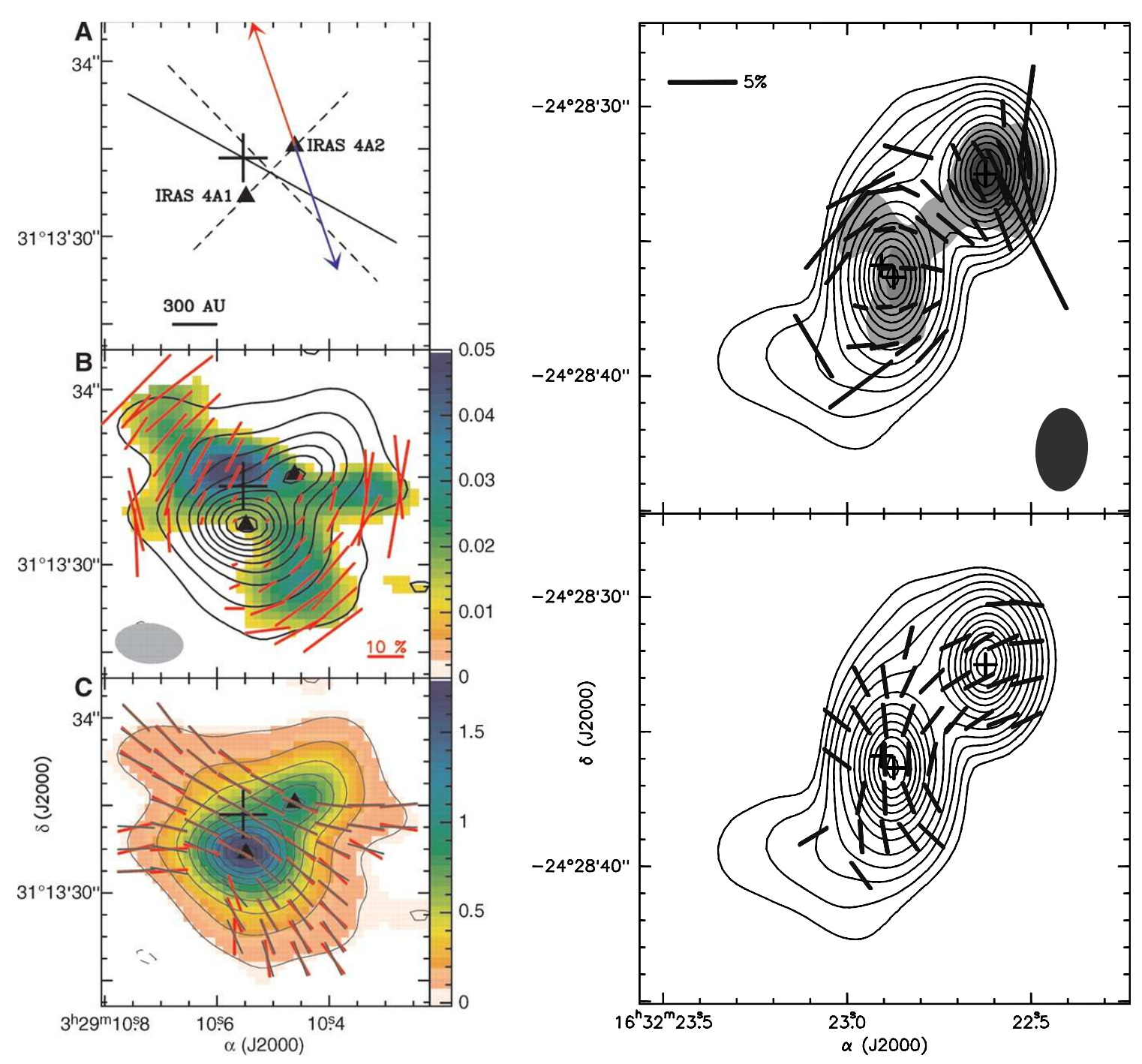

Figure 1: (LEFT) Polarization in NGC 1333 IRAS 4A- (A) Sketch of the axis directions: red/blue arrows show the direction of the redshifted/blueshifted lobes of the molecular outflow, solid lines show the main axis of the magnetic field, and dashed lines show the envelope axes. The solid triangles show the position of IRAS 4A1 and 4A2. The small cross shows the centre of the magnetic field symmetry. (B) Contour map of the $345 \mathrm{GHz}$ dust emission (Stokes I) superposed with the color image of the polarized flux intensity. Red vectors: Length is proportional to fractional polarization and the direction is position angle of linear polarization. The synthesized beam is shown in the bottom left corner. (C) Contour and image map of the dust emission. Red bars show the measured magnetic field vectors. Grey bars correspond to the best fit parabolic magnetic field model. Using the residuals from this fit, the strength of the magnetic field can be estimated with the CF method. (RIGHT) Polarization in IRAS 16293- Top panel: Contour map of the total (Stokes I) dust emission overlaid on the grayscale image of the polarized dust intensity. The gray bars represent the polarization vectors. The 5\% vector length is shown in the top left panel for comparison. Their length is proportional to the polarization fraction. Black crosses mark the position of $\mathrm{Aa}, \mathrm{Ab}$, and $\mathrm{B}$ sources. The synthesized beam is shown in the bottom right. Bottom panel: Contour map of the total dust emission as in the top panel. The bars represent the magnetic field vectors. 

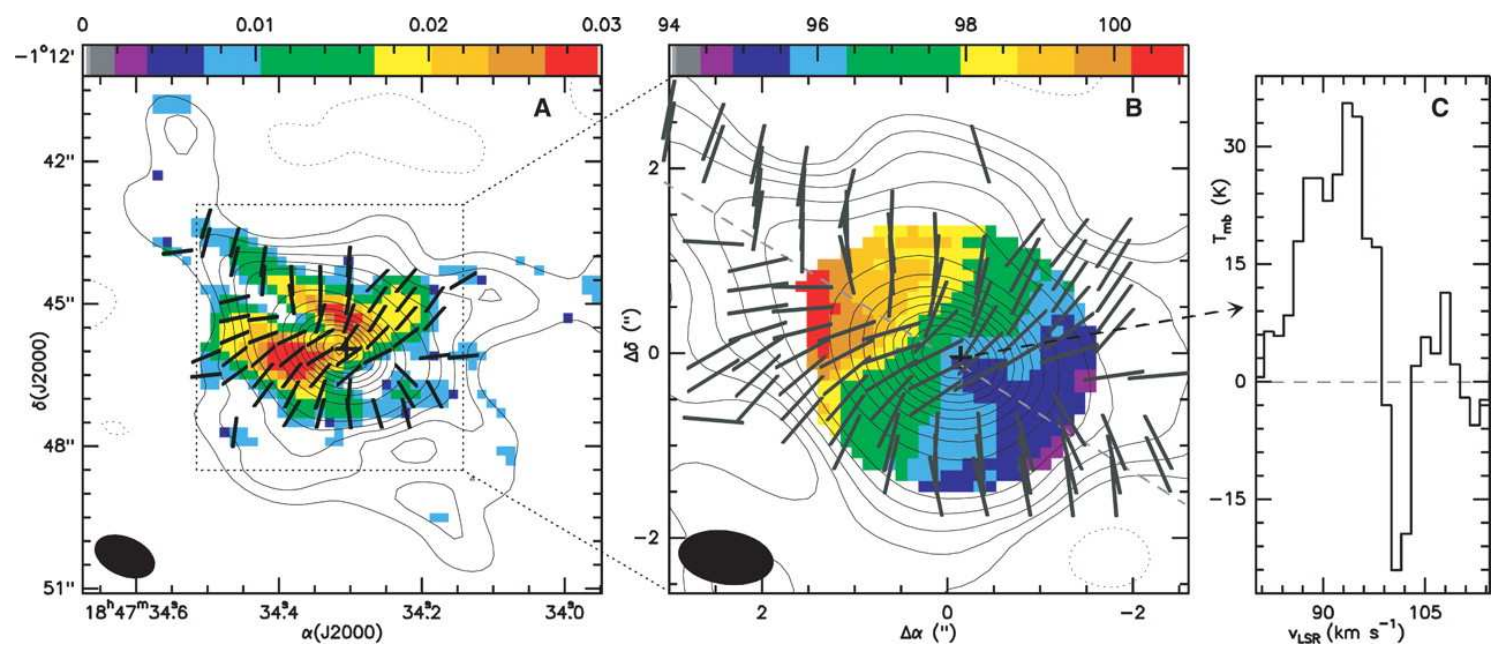

Figure 2: Polarization in G31.41: (A) Contour map of the $345 \mathrm{GHz}$ dust emission superposed on the color image of the polarized flux intensity. Black thick bars indicate the position angle of the magnetic field. The full width half maximum synthesized beam is $1.34^{\prime \prime} \times 0.83^{\prime \prime}$ with a position angle of $67^{\circ}$ (shown in the bottom left corner). (B) Contours of the dust emission superposed on the color image of the flux weighted velocity map of the $\mathrm{CH}_{3} \mathrm{OH}$ 147-156 A. Black thick bars indicate the direction of the magnetic field. (C) Spectrum of the $\mathrm{C}^{34} \mathrm{~S} 7-6$ line at the position of the dust emission peak.

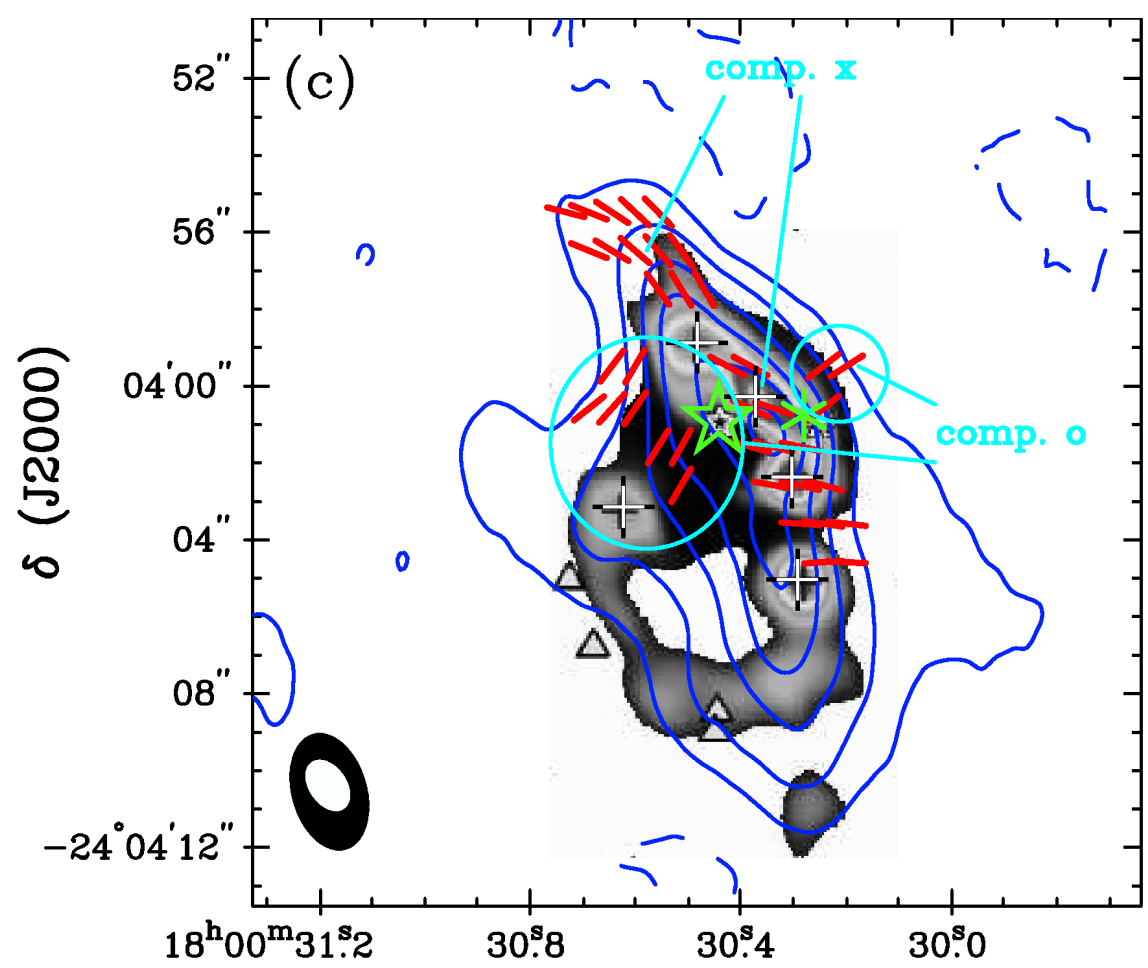

Figure 3: Polarization of the continuum emission in G5.89: In this figure, the solid contours represent the total dust continuum emission (Stokes I). The grey scale image shows the map obtained with higher resolution (Hunter et al. 2008). The length of the red vectors is proportional to the fractional polarization while the direction represents the direction of the magnetic field. The two components " $\mathrm{x}$ " and " 0 " of the B-field likely arise from different origins as discussed in the text. 

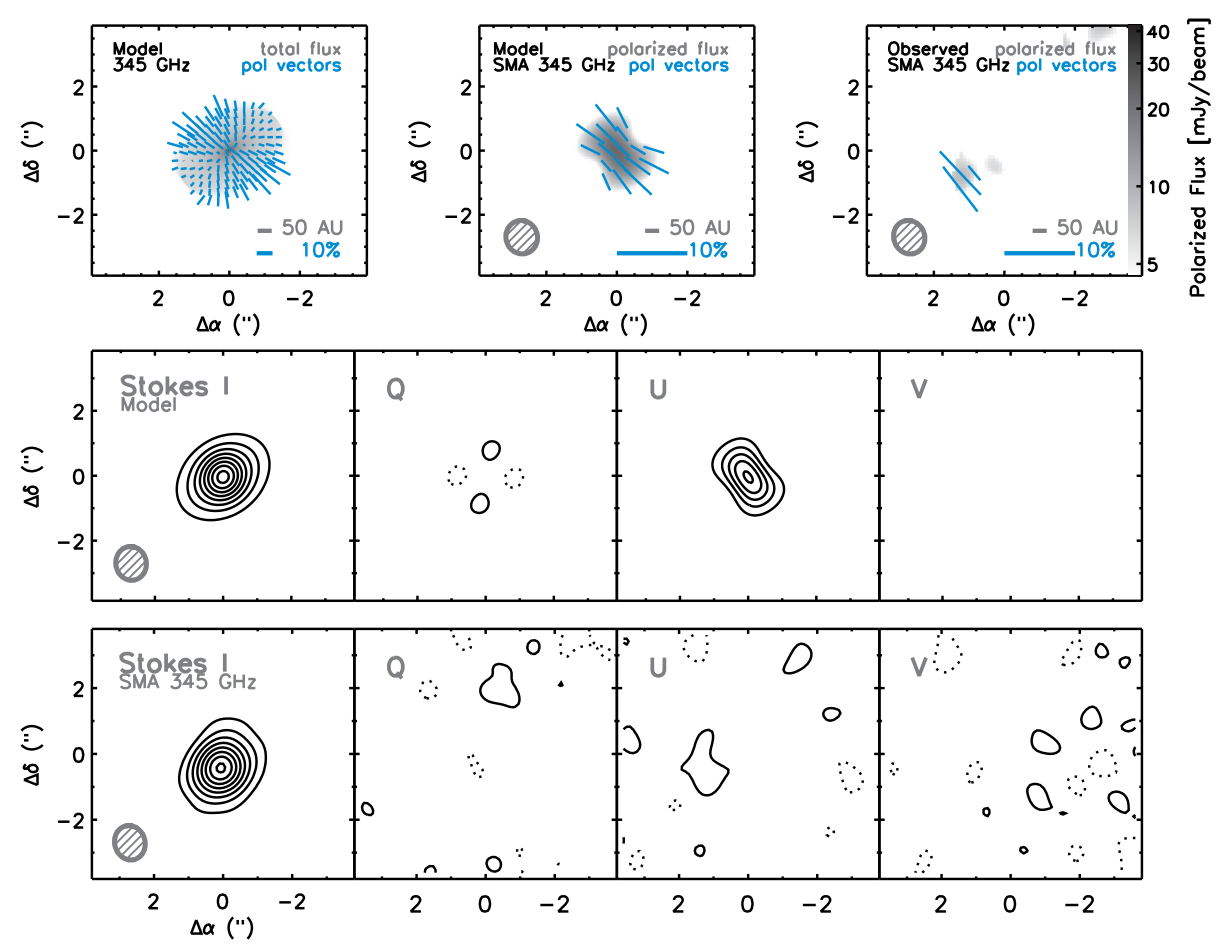

Figure 4: Comparison between the Cho et al., (2007) model and the SMA $340 \mathrm{GHz}$ observations of HD 163296. The top row shows the prediction for the model at full resolution (left), a simulated observation of the model with the SMA (center), and the 2008 SMA observations (right). The grayscale shows either the total flux (left) or the polarized flux (center, right), and the blue vectors indicate the percentage and direction of polarized flux at half-beam intervals. The center and bottom rows compare the model prediction (center) with the observed SMA data (bottom) in each of the four Stokes parameters (I, Q, U, V, from left to right). 